\title{
Effects of Gas Rarefaction on Used Nuclear Fuel Cladding Temperatures during Vacuum Drying
}

\author{
Hadj-Nacer, M. ${ }^{* 1}$, Manzo, T. ${ }^{1}$, Ho, M. T. ${ }^{2}$, Graur, I. ${ }^{2}$ and Greiner, M. ${ }^{1}$ \\ ${ }^{1}$ University of Nevada Reno, 1664 N. Virginia St. Reno, 89557 Nevada, USA. \\ ${ }^{2}$ Aix-Marseille Université, CNRS UMR 7343, 13453 Marseille, France \\ *mhadjnacer@unr.edu
}

A two-dimensional computational model of a loaded used nuclear fuel canister filled with dry helium gas was constructed to predict the cladding temperature during vacuum drying conditions. The model includes distinct regions for the fuel pellets, cladding and helium within each basket opening, and it calculates conduction heat transfer within all solid components, heat generation within the fuel pellets, and conduction and surface-to-surface radiation across the gas-filled regions. First steady-state simulations are performed to determine peak clad temperatures as a function of fuel heat generation rate, assuming the canister is filled with atmospheric-pressure helium. The allowable fuel heat generation rate, which brings the peak clad temperature to its limit is evaluated. The discrete-velocity-method is then used to calculate slip-regime rarefied-gas conduction across planar and cylindrical helium-filled gaps. These results are used to verify the Lin-Willis solid/gas interface thermal-resistance model for a range of thermal accommodation coefficients, $\alpha$. The Lin-Willis model is then implemented at the solid/gas interfaces within the canister model. Finally, canister simulations with helium pressures of 100 and $400 \mathrm{~Pa}$ and $\alpha=1,0.4$ and 0.2 are performed to determine how much hotter the fuel cladding is under vacuum drying conditions compared to atmospheric pressure. For $\alpha=0.4$, the fuel heat generation rates that bring the clad temperature to its allowed limit for helium pressures of 400 and $100 \mathrm{~Pa}$ are reduced by $10 \%$ and 25\%, respectively compared to atmospheric-pressure conditions. Transient simulations show that the cladding reaches it steady state temperatures roughly 20 to 30 hours after water is removed from the canister.

\section{Introduction}

Pressurized water reactor (PWR) nuclear fuel assemblies consist primarily of $14 \times 14$ to $18 \times 18$ square arrays of Zircaloy cladding tubes that contain fuel pellets and high-pressure gases [1]. During reactor operations, the fuel pellets become highly radioactive and form fission-product gases. After being discharged, Used Nuclear Fuel (UNF) assemblies are stored underwater while their radioactivity and heat generation rate decrease [2]. After sufficient time, a canister with an internal basket is placed in a transfer cask and lowered into the pool. The canister is then loaded with used assemblies, covered, and lifted out of the pool. Helium or another non-oxidizing gas is forced into a port near the top of the canister while water flows out through a tube that reaches to the canister bottom [3]. Small amounts of water may remain at the bottom and in crevices of the canister, basket and assembly surfaces after draining. Essentially all moisture must be removed to prevent corrosion of the assembly and cask materials, and/or formation of combustible mixtures of hydrogen and oxygen [4]. After the drying operation, the canister is filled with helium to pressures up to $7 \mathrm{~atm}(710 \mathrm{kPa})$ and sealed. It is then placed in an air natural-convection cooled onsite storage module or another packaging for offsite transport.

With the absence of a defined used-fuel disposal and/or reprocessing path, it is crucial to assure the safety of long-term dry storage systems [5]. Federal regulations (10CFR72) require that these systems ensure that external radiation doses are below certain limits, and that the fuel configuration remains subcritical, confined and contained, and retrievable. Federal regulations (10CFR71) also require that transport package performance be analyzed under normal conditions of transport (which include a $0.3-\mathrm{m}$ drop) and hypothetical accident conditions (which include a 9-m drop). The cladding holds the used fuel pellets and fission gas in their analyzed configuration. Adequate cladding ductility must be maintained to assure that, after decades in storage, the assemblies can be safely transferred to other packages and/or transported to other locations in their "as analyzed" configuration. 
Radial hydride formation within the cladding has the potential to radically reduce its ductility and suitability for long-term storage or transport [5]. During all post-reactor drying, transfer, storage and transport operations the fuel cladding must be kept below certain temperatures to avoid (a) dissolution of circumferential hydrides that exist in the cladding, and (b) high gas pressures within the tubes, which leads to high cladding hoop stress [6]. If this occurs, then as the heat generation of the used fuel decreases during long-term storage, radial hydrides may precipitate and cause the cladding to become brittle [7-10]. The operations in which the fuel canister is dried [11], moved to an onsite storage facility, and first placed in a storage module are of concern because they are the first in which the fuel is removed from water-cooled environments, and its heat generation rate may still be relatively high. This may cause the cladding to reach high temperatures and trigger events that can lead to radial hydride formation.

Nuclear Regulatory Commission Interim Staff Guidance-11, Revision 3 (ISG-11) [6] specifies conditions that are intended to prevent radial hydride formation. For example, the maximum calculated fuel cladding temperature must remain below $400^{\circ} \mathrm{C}$ for normal conditions of storage and short term loading conditions (e.g., drying, backfilling with inert gas, and transfer of the cask to the storage module). For low burnup fuel, a higher short-term temperature limit may be used, provided that the best estimate of the cladding hoop stress is less than $90 \mathrm{MPa}$. During loading operations, repeated temperature cycling is allowed, but is limited to less than 10 cycles in which the cladding temperature varies by more than $65^{\circ} \mathrm{C}$. Until further guidance is developed, high burnup fuel will be handled on a case-by-case basis [6]. In Germany and Japan the maximum cladding temperatures for storage and drying are lower and equal to, respectively, $370^{\circ} \mathrm{C}[12]$ and $250^{\circ} \mathrm{C}[13]$.

Canister Drying Operations Two methods are currently used by industry for canister moisture removal: vacuum drying and forced helium dehydration $[3,6]$. In vacuum drying, the canister is evacuated to pressures as low as $67 \mathrm{~Pa}(0.5$ Torr) to promote evaporation and water removal [11]. Several cycles of evacuation and refill may be necessary before operators can demonstrate that the canister meets the drying technical specification of maintaining a low pressure of $400 \mathrm{~Pa}$ (3 Torr) for 30 minutes [3,11]. This process requires roughly 12 to 24 hours for canisters containing metal matrix baskets, and 60 to 100 hours if BORAL ${ }^{\circledR}$ is used as a Neutron absorber [13].

At the low gas densities associated with vacuum drying, buoyancy-induced gas motion and natural convection heat transfer within the helium-filled regions of the canister are essentially eliminated. Helium thermal conductivity is nearly the same at vacuum-drying pressures as it is at atmospheric conditions. However, as the gas pressure decreases, the mean free path length between molecular collisions (described later in this paper) increases. When it approaches the characteristic dimension of the enclosure containing the gas the gas becomes rarefied. In general, rarefied gases act as a vast collection of discrete colliding molecules that may be modeled using the Boltzmann equation [14, 15], rather than a continuum whose transport can be described using the Navier-Stokes and Fourier equations. Gas rarefaction reduces its ability to conduct heat compared to a continuum. As a result, if vacuum drying causes rarefaction, then the fuel cladding will be hotter than it would be under atmospheric-pressure conditions.

Forced helium dehydration is used for drying canisters containing high-burnup and other high heat generating fuel [3]. In that process, helium is forced to circulate through the canister, and moisture is removed from the gas while it is outside by condensing, demoisturizing, and then preheating. The gas pressure during helium dehydration is maintained at roughly 3 and 4 atm (304 to $405 \mathrm{kPa}$ ) [16], so natural convection is active and, based on the dimension of the canister gaps containing the gas, the gas is not rarefied. Fuel cladding temperatures may be lower when forced helium dehydration is used compared to vacuum drying. However, gas condensing and demoisturizing equipment are required in the former process, but not needed for the latter.

Cladding Temperature Prediction Currently, package vendors predict cladding temperatures and the resulting hoop stresses during drying using experimentally-benchmark whole-package Computational Fluid Dynamics (CFD) simulations [3, 17]. In some models, the fuel and basket are replaced by a smeared region with an effective thermal conductivity and an effective porosity. Other models use an accurate-geometry computational domain where the fuel rods, gas and the baskets are distinctly modeled [18-21]. These models are used to predict the peak cladding temperature for a range of fuel heat generation rates. That 
information is used to find the fuel heat generation rate that causes the clad temperature to reach its allowed limits. It may also help determine which loaded canisters may be vacuum-dried, and which must use the more complex forced-helium-dehydration process.

The whole-package computational models have been validated [22] against measurements performed in an actual evacuated storage package [23]. Currently, the effective properties used during vacuum drying are calculated without regard to gas rarefaction. However, the fuel heat generation in the tests used to validate the current methods was moderately low. For a given package, the effect of gas rarefaction on peak cladding temperatures increases with fuel heat generation rate. Rarefaction effects may need to be included for high-burnup and other high-heat-generating fuels.

Current Work The objective of the current work is to develop computational methods that can be used to predict cladding temperatures under vacuum drying conditions, including the effects of gas rarefaction. In this work, a finite difference cross-sectional model of a canister designed for 24 PWR assemblies, similar to the TN24P NUHOMS® Horizontal Modular Storage System [24, 25], is constructed using ANSYS/Workbench. It accurately represents $15 \times 15$ arrays of fuel rods and helium gas within each basket opening and a helium-filled gap between the basket and canister surface. Steady-state simulations that model conduction in all components and surface-to-surface radiation across the helium-filled regions are performed to predict the peak cladding temperature for a range of fuel heat generation rates.

Initial simulations are performed by modeling the helium gas as a continuum at a pressure of $10^{5} \mathrm{~Pa}$ $(\sim 1 \mathrm{~atm})$. Boltzmann equation simulations are then performed to predict conduction heat transfer across rarefied helium within simple gaps between parallel plates and concentric cylinders. These calculations use the Shakhov model (S-model) kinetic equation [26, 27]. These results are used to assess the accuracy of a mildly-rarefied-gas conduction heat transfer model. This model employs a continuum model within the bulk of the gas, and the Lin-Willis temperature-jump model [28] at the gas/solid interfaces to include rarefaction effects. Based on the quantitative agreement between these models, the Lin-Willis temperaturejump model is then implemented in the ANSYS canister simulation. It is used to predict the increased cladding temperatures caused by gas rarefaction at pressures of 100 and $400 \mathrm{~Pa}$. The first pressure is chosen because it is close to the lowest pressure used during vacuum drying [11]. The second pressure is used to test if the moisture has been removed from the system.

Another set of simulations is performed to estimate the amount of time after water is removed from the canister for the cladding to reach its steady-state temperature. This is compared to the typical time required to vacuum dry a canister to assess the appropriateness of using steady-state analysis to estimate the peak cladding temperature during drying. To simplify the modeling in this work, dry helium gas is considered throughout. As a result, evaporation and moisture transport are not included, but may be considered in future research.

\section{UNF Canister Computational Model}

In this work we consider a UNF canister with an internal basket, similar to a Transnuclear NUHOMS ${ }^{\circledR}$ TN-24PTH [24, 25] loaded with 24 Westinghouse 15x15 PWR assemblies [29]. Figure 1 shows the twodimensional computational model of the canister cross-section employed in this work. The model takes advantage of the canister symmetry along radial-lines, so only one-eighth of its cross section is included. Figure 1a shows the material regions. Each fuel assembly consists of a $15 \times 15$ array of 10.92 -mm outerdiameter rods. Each fuel rod consists of $d=9.58$-mm-diameter $\mathrm{UO}_{2}$ pellets surrounded by $0.67-\mathrm{mm}$ thick zircaloy cladding. There are also thirteen hollow zircaloy tubes in each assembly. The rod center-to-center pitch is $14.43 \mathrm{~mm}$, and the distance between the walls and the nearest rod center is $12.04 \mathrm{~mm}$. The square cross-section tubes that line each basket opening are constructed from stainless steel, and some surfaces are backed by BORAL ${ }^{\circledR}$ neutron poison plates. At this cross section, the tubes are supported by an aluminum structure. The basket and assemblies are enclosed within a stainless steel canister, and there is a large void space in the upper right of the domain. The void spaces contain dry helium. 
Figure $1 \mathrm{~b}$ is a detailed view of one corner of an assembly within a stainless steel basket opening, a BORAL $^{\circledR}$ plate, and the aluminum support. Figure 1c shows a region at the periphery of the canister, including a 2.29-mm-wide helium-filled gap between the basket and canister wall. Figure 1d shows the computational mesh within the region shown in Fig. 1b. The entire two-dimensional computational mesh has 131,202 elements.

The characteristic dimension of the canister's helium-filled spaces is needed to compare with the gas mean free path length in order to determine if the gas is rarefied. However, for the complex space in Fig. 1 this dimension is not easily defined. In this model, the minimum surface-to-surface spacing is $2.3 \mathrm{~mm}$ between the outer basket and inner canister surfaces. The maximum spacing is $9.5 \mathrm{~mm}$ between diagonallyadjacent fuel rods.

Steady-state and transient thermal simulations were performed using ANSYS/Fluent 15, which has been benchmarked for configurations relevant for used fuel packaging [18, 19 and 22]. These simulations assume uniform heat generation in all the $\mathrm{UO}_{2}$ regions and include conduction within the solid and helium -filled regions. Surface-to-surface radiation across helium-filled spaces is included, with surface emissivities of 0.46 for the stainless steel, and 0.8 for both the aluminum and zircaloy [30]. These simulations also have the capacity to include a user-defined resistance at all gas/solid interfaces, which is activated when modeling mildly-rarefied helium. During drying operations, the canister is vertical, so that the gravitational field is perpendicular to the plane of Fig. 1. While some natural convection effects may be present in the helium regions, they are not included in these simulations. This is because the gravitational vector is perpendicular to the plane of Fig. 1, and so the primary flow direction can not be included in the two-dimensional computational model.

At the package axial location that is represented by this model, the volumetric heat generation within all the $\mathrm{UO}_{2}$ pellets is assumed to be uniform and equal to

$$
q=\frac{P_{F} Q_{F}}{N L \frac{\pi}{4} d^{2}} .
$$

In this expression, $Q_{F}$ is the fuel assembly total heat generation rate, $N=212$ is the number of fuel rods within each assembly containing $\mathrm{UO}_{2}$ pellets, and $L=3.66 \mathrm{~m}$ is the fuel rod length that contains pellets, based on a $15 \times 15$ Westinghouse PWR assemble [29]. The peaking factor $P_{F}=1.1351$ [32] is the heat generation rate at the axial location where it reaches its maximum level divided by the average for the assembly.

For all steady-state and transient simulations reported in this paper, symmetry boundary conditions (insulated and radiatively-reflective) are applied along the radial lines in Fig. 1a. During drying operations, cooling water is circulated in the gap between the canister outer surface (curved surface in Fig. 1a) and transfer cask inner surface to reduce temperatures. Throughout this work, the canister outer boundary surface temperature is assumed to be $101.7^{\circ} \mathrm{C}$ [31] to conservatively model contact with boiling water at the highest possible pressure during the drying operation.

\section{Continuum-Gas Heat Transfer within a Canister}

This section presents continuum-gas simulations. These simulations model the canister filled with roughly atmospheric-pressure dry helium (pressure $P=101,325 \mathrm{~Pa} \sim 10^{5} \mathrm{~Pa}$ ), do not include the effect of gas rarefaction, and are used to compare to the rarefied-gas results. Figure 2 shows continuum-model temperature contours for a fuel assembly heat generation of $Q_{F}=1498 \mathrm{~W} /$ assembly. Outlines of the package components are also shown. There are local temperatures maximums within each fuel assembly, but the global peak is located near the center of the innermost assembly, on the upper domain boundary. Figure 2 shows the $r$-axis along that boundary with its origin at the package center. The maximum or peak 
cladding temperature for this fuel heat generation rate is $T_{P}=261^{\circ} \mathrm{C}$, which is below the nominal allowable limit value used in the United States, $T_{L}=400^{\circ} \mathrm{C}$.

The lower solid line in Fig. 3 marked "Continuum" shows the temperature profile along the $r$-axis for the conditions used in Fig. 2. The temperature exhibits local peaks within the two fuel assemblies through which the $r$-axis passes, but the inner assembly temperature is roughly $25^{\circ} \mathrm{C}$ warmer than the outer one. Within the fuel assemblies, the temperature profile exhibits a stair-step shape. The more isothermal regions are within the relatively-high thermal-conductivity solid components. The lower-conductivity gas exhibits steeper gradients. The very steep gradient at $r=83-84 \mathrm{~cm}$ is within the helium-filled gap shown in Fig. 1c. This gradient is particularly steep because the heat flux through that region is relatively high, and the contribution of radiation to the total heat transfer across this gap is relatively low due to its low temperatures. This region makes a significant contribution to the total temperature difference between the canister outer surface and the hottest cladding.

The upper solid line in Fig. 3 marked "Hard Vacuum" shows the temperature profile along the $r$-axis assuming the helium is completely evacuated and $Q_{F}=1498 \mathrm{~W} /$ assembly. This eliminates conduction across all void spaces, so all heat transfer in those regions is by radiation. The shape of this profile is nearly identical with that of the continuum simulation. The primary difference is that the temperature gradient in the gap between the basket outer surface and the canister inner surface is much greater for the hard vacuum than for the continuum simulation. We define the basket surface temperature increase $\Delta T_{B S}$ as the outer basket surface temperature (at $r=83 \mathrm{~cm}$ ) at low pressure, minus its value under continuum conditions. For the hard vacuum simulation $\Delta T_{B S}=142.4^{\circ} \mathrm{C}$. The peak clad temperature increase $\Delta T_{P C}$ is the peak clad temperature at low pressure minus its value for continuum conditions. For the hard vacuum simulation $\Delta T_{P C}=143.5^{\circ} \mathrm{C}$, which is only $1.1^{\circ} \mathrm{C}$ larger than $\Delta T_{B S}$ (these results are included in Table 1). This indicates that the effect of eliminating conduction in the void spaces within the basket openings is much smaller than it is in the gap between the basket and canister. This is because radiation heat transfer caries a larger fraction of the heat within the basket, since that region is relatively hot, compared to the gap between the canister and basket, which is relatively cool.

The solid lines in Fig. 4 shows the maximum or peak cladding temperatures predicted by the continuum and hard vacuum models as functions of the assembly fuel heat generation. Horizontal lines show the nominal fuel clad temperature limits used in the United States and in Germany $T_{L}=400^{\circ} \mathrm{C}$ [6] and $370^{\circ} \mathrm{C}$ [12], respectively. The continuum and hard vacuum simulations indicate that the fuel cladding will reach $400^{\circ} \mathrm{C}$ when the fuel heat generation rate reaches limit values of, respectively, $Q_{F L}=3202$ and 1461 W/assembly (54\% smaller). These limit heat generation rates $Q_{F L}$ are included in Table 2. Table 2 also shows the values of $Q_{F L}$ if the lower temperature limit of $370^{\circ} \mathrm{C}$ is used and indicates that $Q_{F L}$ is reduced by $12-16 \%$.

We note that the Certificate of Compliance of the NUHOMS ${ }^{\circledR} 24 \mathrm{P}$ system $[24,25]$ requires the average fuel heat generation rate be less than $1000 \mathrm{~W} /$ assembly for a standard horizontal storage module, or 1700 $\mathrm{W} /$ assemble for an enhanced module. These heat generation rates are lower than the limit values presented in Table 2 for atmospheric pressure helium. For the purposes of this paper, we assume that the Certificate of Compliance limit is calculated by the manufacturer to maintain the cladding safely below $400^{\circ} \mathrm{C}$ during storage, in which the canister is cooled by air natural convection.

Calculation of cladding temperatures under dry storage conditions is beyond the scope of this work. However, it is reasonable that the canister surface is hotter in the storage module, where it is cooled by air, than in vacuum drying, where it is submerged in water. If the canister is filled with atmospheric pressure helium in both situations, then it is also reasonable that the cladding temperature may be hotter in the storage module than during vacuum drying. However, including the effects of rarefication during vacuum drying increases the predicted cladding temperature under that condition.

\section{Rarefied Gas Heat Transfer across Simple Gaps}


We now consider the physical processes that affect conduction heat transfer across rarefied gases, and models for predicting that transport. The molecular mean free path length $\lambda$ of a gas is defined as the average distance molecules travel between consecutive collisions. Based on kinetic theory and the hard sphere intermolecular interaction model for a pure gas with molecule mass $m$, the mean path length is [33]

$$
\lambda=\frac{\sqrt{\pi}}{2} \frac{\mu}{P} \sqrt{\frac{2 k_{B} T}{m}} .
$$

In this expression $k_{B}$ is Boltzmann's constant, and the gas pressure, temperature and dynamic viscosity are $P, T$ and $\mu$, respectively. The temperature-dependent viscosity may be calculated based on the hard sphere intermolecular interaction model as

$$
\mu=\mu_{0}\left(\frac{T}{T_{0}}\right)^{1 / 2}
$$

where $T_{0}=273.15 \mathrm{~K}$ and $\mu_{0}$ are the gas' reference temperature and viscosity. For helium $\mu_{0}=1.865 \times 10^{-5}$ $\mathrm{Pa} \cdot \mathrm{s}$. Combining these results indicates that the mean free path length is proportional to the temperature and inversely proportional to the pressure, both to the first power.

If a gas is contained within an enclosure of characteristic length $L_{C}$, and if the molecular mean free path length is sufficiently small, then the gas behaves as a continuum and its momentum and thermal transport is accurately modeled using the Navier-Stokes and Fourier equations. The Knudsen number is defined as the ratio of the molecular mean free path to the enclosure characteristic length, $K n=\lambda / L_{C}$. The continuum approximation is generally valid for $K n<10^{-3}$ [14]. If the gas pressure is decreased while the characteristic length is kept the same such that $K n>10^{-3}$, the gas no longer behaves as a continuum. The rarefied gas may be modeled as a vast collection of molecules that collide with each other and the enclosure walls. The Boltzmann equation may then be used to calculate transport, but its numerical solution is computationally intensive, especially in complex enclosures.

Whenever an individual molecule collides with a wall, its energy level can remain the same as it was before the collision. Alternately, it can fully-accommodate the wall conditions, so that its post-collision energy is in equilibrium with the wall temperature. For a given gas (collection of molecules) and solid surface, the thermal accommodation coefficient $\alpha$ is defined as the fraction of molecules that accommodate the wall conditions. It can be related to the temperatures of the collections of incident and reflected molecules $T_{i}$ and $T_{r}$, and the temperature of the wall $T_{w}$, as [34]

$$
\alpha=\frac{T_{i}-T_{r}}{T_{i}-T_{w}}
$$

The thermal accommodation coefficient is zero if all of the reflected molecules have the same energy they had when they approached the wall, unity if all reflected molecules have the same temperature as the wall, or some value between 0 and 1 .

In general, the accommodation coefficient can be a function of the gas and wall compositions, and the wall surface topography and contamination [35, 36]. For "engineering surfaces," with typical surface contamination, the value of $\alpha$ is primarily dependent on gas compositions and temperature, and is essentially independent of the wall properties [37]. For example, for helium gas it varies from 0.4 to 0.2 as the temperature increases from 300 to $700 \mathrm{~K}$. In general, the value of $\alpha$ is higher for gases with heavier molecular masses than for lighter ones. 
In a given enclosure, as the gas pressure goes down and the mean free path length increases, the frequency of molecular-molecular collisions is reduced compared to molecular-wall interactions. As a result, the relative importance of molecular-surface interactions increases. For gases in the continuum regime, energy transfer to the walls is not affected by $\alpha$, because molecular-wall interactions are relatively unimportant. However, as the pressure decreases and the gas becomes rarefied, the importance of molecular-wall interaction increases, and $\alpha$ affects heat transfer across the gas.

Graur et al. [26, 27] used the Shakhov kinetic equation as a model (S-model) to simplify the collisional term of the full Boltzmann equation. In the current work, we numerically solve the S-model kinetic equation within two simple enclosures using the Discrete Velocity Method (DVM). While the specifics of this calculation is not included in this paper, they may be found in [26, 27]. Figures $5 \mathrm{a}$ and $5 \mathrm{~b}$ show simple gas-filled computational domains between, respectively, parallel plates and concentric cylinders. In both configurations $T_{A}=330 \mathrm{~K}$ and $T_{B}=300 \mathrm{~K}$, and the $y$-coordinate systems are shown. The planar wall-towall spacing is $L_{C}=10 \mathrm{~mm}$, and the inner and outer radii of the annular gap are respectively $R_{A}=5 \mathrm{~mm}$ and $R_{B}=10 \mathrm{~mm}\left(L_{C}=R_{B}-R_{A}=5 \mathrm{~mm}\right)$. In this work the Maxwellian specular-diffuse boundary conditions, with $\alpha=1$, were implemented at the walls [38], and the hard sphere intermolecular interaction model was used for the viscosity calculation (Eq. 3).

Figures $6 \mathrm{a}$ and $6 \mathrm{~b}$ show the temperature $T$ versus $y$ in the planar and cylindrical domains for helium gas and a thermal accommodation coefficient of $\alpha=1$. In each plot, results are shown for four pressures $P$ corresponding to $K n=0.001,0.01,0.1$ and 1 (the dimensional pressure for each simulation is shown in parentheses). The solid lines show the S-model kinetic simulation results. In both configurations, for the lowest Knudsen number $K n=0.001$ (highest gas pressure) the gas temperature at the wall $T_{g}$ is essentially the same as the wall temperature $T_{w}$. However, at higher $K n$ (lower pressures) there is a temperature difference between the gas and wall (temperature-jump), and this jump increases as the pressure decreases. S-model simulation results at the same pressures but smaller values of $\alpha$ (not included in Fig. 6) have larger temperature jumps.

Analysis shows that transport across mildly-rarefied gas-filled gaps, i.e. $10^{-3}<K n<10^{-1}$ may be accurately calculated using a continuum model (Navier-Stokes and Fourier equations) completed with the temperature-jump conditions at the gas/solid interfaces [39]. This analysis predicts that the difference between the gas and wall temperatures $T_{g}$ and $T_{w}$ is proportional to the heat transfer rate conducted by the gas away from the wall $Q$, and may be calculated as:

$$
T_{g}-T_{w}=R_{T J} Q .
$$

In this expression, $R_{T J}$ is the temperature jump thermal resistance at the gas/surface interface, and $Q$ does not include the heat transported by radiation to other surfaces. The temperature-jump thermal-resistance is

$$
R_{T J}=\frac{\lambda \zeta_{T}}{A \kappa},
$$

where $A$ is the boundary surface area, $\kappa$ is the gas thermal conductivity, and $\zeta_{T}$ is the temperature jump coefficient. The gas conductivity is related to its viscosity $\mu$ (Eq. 3), gas specific heat at constant pressure $c_{P}$ and Prandtl number Pr as

$$
\kappa=\frac{c_{P}}{\operatorname{Pr}} \mu
$$

For helium, the specific heat and Prandtl number are constants and equal to $c_{p}=5193 \mathrm{~J} / \mathrm{kgK}$, and $\operatorname{Pr}=2 / 3$.

The literature contains numerous models for the temperature jump coefficient. Lin and Willis [26] developed the following expression that is based on a solution of the Bhatnagar-Gross-Krook (BGK) kinetic model equation, and is dependent on $\operatorname{Pr}, \alpha$, and the gas ratio of specific heat $\gamma$, 


$$
\zeta_{T}=\frac{2 \gamma}{(\gamma+1) \operatorname{Pr}}\left(\frac{2-\alpha}{\alpha}+0.17\right)
$$

For helium $\gamma=5 / 3$. This expression shows that the jump coefficient increases as $\alpha$ decreases.

The dashed lines in Fig. 6 show temperature profiles calculated using the ANSYS/Fluent CFD package in the domains shown in Fig. 5. Those simulations employed a continuum model in the bulk of the gas, with the temperature-dependent gas thermal conductivity in Eqs. (3 and 7), and the temperature-jump thermal resistance at the gas/solid interfaces (Eq. 6) with the Lin-Willis temperature jump coefficient (Eq. 8 ). For the planar and cylindrical configurations, these profiles exhibit excellent agreement with the DVMcalculations for the $K n=0.001,0.01$ and 0.1 (the highest three pressures), but diverge at $K n=1$ (lowest pressure). These Knudsen numbers are consistent with the temperature-jump regime, i.e. $10^{-3}<K n<10^{-1}$ [14].

Figure 7 shows the dimensionless heat transfer reduction ratio $\left(Q_{C}-Q\right) / Q_{C}$ versus gas pressure for both enclosures in Fig. 5, and $\alpha=1,0.4$ and 0.2. In this expression, $Q$ is the conduction heat transfer between the walls for the given pressure and thermal accommodation coefficient, and $Q_{C}$ is the heat transfer in the continuum regime. The solid and dashed lines show results from, respectively, the S-model kinetic simulations, and the ANSYS/Fluent simulations with the Lin-Willis temperature jump model. The two models are in excellent agreement for the pressure range included in the plots $(P>10 \mathrm{~Pa})$. They show that the heat transfer reduction increases as the pressure decreases (and the gas becomes more rarefied), and as $\alpha$ decreases (and the gas accommodation to the wall decreases). The quality of the agreement decreases as the pressure decreases. Complete comparisons between the Lin-Willis temperature jump model and Smodel kinetic equation results are presented in [41]. The quantitative agreement between the S-model and Lin-Willis temperature jump models justifies implementing the temperature jump model in the domain in Fig. 1 to predict cladding temperatures under moderately-rarefied conditions.

\section{Rarefied-Gas Heat Transfer within a Canister}

As mentioned earlier, Boltzmann equation solutions are needed to calculate temperatures within the canister under rarefied conditions. Those solutions are computationally intensive, particularly in such a complex enclosure. However, for mildly-rarefied gases the effects are concentrated near the gas/solid interfaces. The last section showed that this effect may be accurately modeled in "simple" gaps between parallel plates and concentric cylinders using continuum (Navier-Stokes and Fourier) equations subjected to the Lin-Willis temperature-jump contact resistance at the gas/solid interfaces.

In this section, we implement this temperature-jump thermal resistance at all gas/solid interfaces within the computation domain shown in Fig. 1. These interfaces are at the surfaces of the zircaloy fuel rods, stainless steel basket openings, aluminum basket outer surfaces, and the stainless steel canister. Simulations are performed for a range of fuel heat generation rates $Q_{F}$, and for gas pressures of $P=100$ and $400 \mathrm{~Pa}$, which are near the lowest values experienced during vacuum drying. Correlations show that the thermal accommodation coefficient for helium gas in contact with engineering surfaces decreases from $\alpha=0.4$ and 0.2 as the interface temperature increases from 300 to $700 \mathrm{~K}$ [37]. In this work, simulations are performed for $\alpha=1,0.4$ and 0.2. However in the current model, the value of $\alpha$ is the same for all interfaces (regardless of temperature). In future work, an improved model will be implemented in which the local value of $\alpha$, and its effect on the temperature jump, will be dependent on the local interface temperature.

Temperature contour plots from simulations that use the temperature jump model, a fuel heat generation rate of $Q_{F}=1498 \mathrm{~W}$, and the range of $\alpha$ and $P$ considered in this work are similar to the one shown in Fig. 2 , and are not included in this paper. The dashed lines in Fig. 3 show temperature profiles along the $r$-axis from the Lin-Willis temperature-jump model for $P=100 \mathrm{~Pa}$ and $\alpha=1,0.4$ and 0.2 . These profiles are between the continuum and hard vacuum results. The shapes of the rarefied-gas simulations are very similar to those from the continuum and hard vacuum results. The major dissimilarity is the temperature difference 
across the narrow helium-fill gap between the basket and canister, $r=83$ to $84 \mathrm{~cm}$. This temperature difference is affected by the temperature-jumps at the gas/solid interfaces on either side of the helium-filled gap.

Table 1 summarizes the basket surface temperature difference $\Delta T_{B S}$ and peak cladding temperature difference $\Delta T_{P C}$ for $\alpha=1,0.4$ and 0.2 and $P=100$ and $400 \mathrm{~Pa}$. As described earlier these are the increase of these temperatures compared to continuum simulation results caused by reduced pressure. Table 1 shows that these temperature differences increase as the accommodation coefficient and the pressure decrease. Moreover, the value of $\Delta T_{P C}$ is at most a few degrees larger than $\Delta T_{B S}$. This indicates that the effect of rarefaction is much smaller in the gas-filled regions within the basket openings than it is in the gap between the basket and canister. This is because the heat flux in the basket opening is small compared to the flux at the basket exterior. Also, radiation heat transfer caries a larger fraction of the heat within the basket than it does near the canister, due to the temperatures of these regions.

The dashed lines in Fig. 4 show the peak clad temperature versus fuel heat generation rate from the rarefied gas simulations. These lines are not parallel to the continuum simulation result, which shows that the difference between the peak clad temperature under rarefied and continuum conditions increases slightly with the fuel heat generation rate. Table 2 summarizes the maximum allowable heat generation rates $Q_{F L}$ (the values of $Q_{F}$ in Fig. 4 that cause the peak clad temperature to reach $T_{L}$ ) for each pressure, thermal accommodation coefficient and cladding limit temperature considered in this work. It indicates that for $T_{L}$ $=400^{\circ} \mathrm{C}$ and $P=100 \mathrm{~Pa}$, the maximum allowable heat generation rates are $11 \%, 25 \%$ and $35 \%$ smaller for $\alpha=1,0.4$ and 0.2 , compared to continuum conditions. For $P=400 \mathrm{~Pa}$, the respective reductions are $3 \%$, $10 \%$ and $17 \%$. For $T_{L}=370^{\circ} \mathrm{C}$, the limit fuel heat generation rate $Q_{F L}$ is reduced by 12 to $14 \%$ compared to $T_{L}=400^{\circ} \mathrm{C}$. Even though $Q_{F L}$ for the rarefied gas simulations are less than the values for continuum simulations, they are greater than the average fuel assembly heat generation rates allowed in the standard or enhanced horizontal storage module Certificate of Compliance, 1000 and $1700 \mathrm{~W} /$ assembly [24, 25].

We wish to confirm that the helium-filled void spaces within the canister are actually in the slip flow regime for the conditions considered in this paper. This regime is characterized by the Knudsen number range $0.001 \leq K n=\lambda / L_{C} \leq 0.1$. As mentioned earlier, the characteristic dimension $L_{C}$ of the helium-filled spaces within the canister shown in Fig. 1 are not as easily defined as those of the simple enclosures shown in Fig. 5. The range of surface-to-surface spacing is between 2.3 and $9.5 \mathrm{~mm}$. Moreover, the different gas temperatures and pressures from each simulation affect the mean free path length $\lambda$. For the higher pressure $P=400 \mathrm{~Pa}$, the Knudsen number is in the range $0.01 \leq K n \leq 0.05$, which is well within the slip flow regime. However, for the lower pressure $P=100 \mathrm{~Pa}$, the Knudsen number is in the range $0.03 \leq K n \leq 0.19$. Under those circumstances, the gas may be slightly in the Transitional regime, which is more rarefied than the slip region. However, this depends on the choice of the characteristic length scale used in Kn.

\section{Transient Temperature Response within a Canister}

The analysis to predict cladding temperatures under vacuum drying conditions described so far assumes the fuel and canister reach their steady state temperatures. However, at the beginning of the drying process, the fuel is relatively cool because it was recently removed from a water-filled pool. As mentioned earlier, the vacuum drying process requires between 12 and 100 hours to complete [13]. In this section, we determine if the fuel and basket approach steady state conditions during this time.

To do this, we assume that at the beginning of the drying process the temperature throughout the canister is uniform at $101.7^{\circ} \mathrm{C}$. This temperature is conservatively-based on assuming the water within and outside the canister is boiling. Transient simulations are then performed to calculate the fuel and canister temperatures versus time after the water within the canister is replaced by dry helium. This simplified calculation neglects several aspects of the actual drying process, including the period to drain the liquid water, and vaporization and transport of the remaining liquid. Simulations assuming the void spaces are filled with dry rarefied-helium, with $P=100 \mathrm{~Pa}$ and $\alpha=0.4$, and dry atmospheric pressure helium are performed with $Q_{F}=1498 \mathrm{~W} /$ Assembly. 
Figure 8 shows the peak cladding temperatures versus time from both simulations. The continuum simulation temperature rises quickly for 15 hours. It goes $98 \%$ of its way from its initial to its steady state temperature after 20.3 hours. The rarefied-simulation temperature goes $98 \%$ of the way to its steady state value after 29.6 hours. Rarefication reduces conduction heat transfer in the helium and increases the steady state fuel temperatures, and this increases the transition time. The simulations were repeated using the limit assembly heat generation rates in Table 2, which are $Q_{F}=Q_{F L}=3202$ and $2408 \mathrm{~W} /$ assembly for the continuum and rarefied-gas conditions, respectively. The $98 \%$-transition times for these simulations are 4 to $7 \%$ shorter than they are for the $1500 \mathrm{~W} /$ assembly calculations. Radiation heat transfer transports more energy at the high temperatures associated with the higher heat generation rate, and causes them to reach steady state more quickly. Since these transient times are of the same order as the amount of time required to dry canisters (12-100 hours), we conclude it is reasonable to use steady state simulations to estimate the peak cladding temperature during drying.

\section{Summary}

Currently, cask vendors do not include the effect of gas rarefaction when predicting the temperature of used nuclear fuel cladding during vacuum drying operations. The objective of this work was to determine the effect of gas rarefaction during those operations.

A geometrically-accurate two-dimensional computational mesh of a helium-filled used-nuclear-fuel canister, similar to the NUHOMS®-24PTH was constructed. A model for heat transfer across mildly rarefied helium was developed using a thermal resistance at gas/solid interfaces. This model was based on results from Lin and Willis [28], dependent on the thermal accommodation coefficient $\alpha$, and found to accurately reproduce temperature profiles and heat transfer across simple gaps calculated by the Shakhovmodel $[26,27]$ of the Boltzmann equation. While the value of $\alpha$ actually depends on surface temperature, in the current implementation, it is uniform at all gas/solid interfaces (which may be at different temperatures).

Steady-state simulations were performed to predict the peak cladding temperature for a range of fuel heat generation rates, under both continuum conditions (no interface resistance), and for mildly rarefied helium at $P=100 \mathrm{~Pa}$ and $400 \mathrm{~Pa}$ and $\alpha=1,0.4$ or 0.2 . For atmospheric pressure (continuum) helium, the fuel heat generation rate that brings the peak cladding temperature to $400^{\circ} \mathrm{C}$ is $Q_{F L}=3202 \mathrm{~W} /$ assembly.

The effect of rarefaction was mostly concentrated in the narrow gap between the basket and canister walls. If the helium is rarefied at $100 \mathrm{~Pa}$, for $\alpha=1,0.4$ or $0.2, Q_{F L}$ is reduced by, respectively, $11 \%, 25 \%$ or $35 \%$, For a helium pressure of $400 \mathrm{~Pa}$, the respective reductions are $3 \%, 10 \%$ and $17 \%$. However, even under these rarefied conditions, the heat generation rates that bring the peak cladding temperature to $400^{\circ} \mathrm{C}$ during vacuum drying are larger than that allowed by the canister's Certificate of Compliance. A transient simulation shows that the cladding temperature approaches steady state conditions between 20 and 30 hours after water is removed from the package. This indicates that steady state simulations accurately predict clad temperatures at the end of canister drying processes that require 12 to 100 hours to complete. The current work did not include the effects of water vaporization or transport during the drying process. These phenomena may be considered in future research.

\section{Acknowledgement}

This work was supported by the US Department of Energy Office of Nuclear Energy University Program. The authors thank the Mesocentre of Aix-Marseille University for the supercomputing resources under the project number $13 \mathrm{~b} 015$. 


\section{REFERENCES}

1. U.S. Dept. of Energy, Office of Civilian Radioactive Waste Management (OCRWM), 1987, "Characteristics of Spent Nuclear Fuel, High-Level Waste, and Other Radioactive Wastes Which May Require Long-Term Isolation," DOE/RW-0184, December.

2. Saling, J.H., and Fentiman, A.W., 2002, Radioactive Waste Management, $2^{\text {nd }}$ Edition, Taylor and Francis, New York.

3. Holtec International, 2010, “HI-STORM 100 FSAR,” Revision 8, Report HI-2002444.

4. Colmont, D. and Roblin, P., 2008, "Improved thermal modeling of SNF shipping cask drying process using analytical and statistical approaches," Packaging, Transport, Storage \& Security of Radioactive Material, Vol. 19, No. 3, pp. 160-164.

5. Hanson, B., Alsaed, H., Stockman, C., Enos, D., Meyer, R., and Sorenson, K., 2012, "Gap Analysis to Support Extended Storage of Used Nuclear Fuel," FCRD-USED-2011-000136 Rev. 0, U.S. Department of Energy Used Fuel Disposition Campaign.

6. NRC, 2003, "Cladding Considerations for the Transportation and Storage of Spent Fuel," ISG-11 R3, Interim Staff Guidance Report for the Spent Fuel Project Office of the U.S. NRC.

7. Daum, R.S., Majumdar, S., Liu, Y.Y., and Billone M.C., 2006, "Radial-hydride Embrittlement of Highburnup Zircaloy-4 Fuel Cladding,” J. Nucl. Sci. Tech., Vol. 43, No. 9, Paper 43090, pp. 1-14.

8. Daum, R.S. Majumdar, S., and Billone, M.C., 2008, "Experimental and Analytical Investigation of the Mechanical Behavior of High-Burnup Zircaloy-4 Fuel Cladding," J. ASTM Intern., Vol. 5, No. 5, Paper ID JAI101209, available online at www.astm.org.

9. Billone, M.C., Burtseva, T.A., and Einziger, R.E., 2013, "Ductile-to-brittle transition temperature for high-burnup cladding alloys exposed to simulated drying-storage conditions," J. Nucl. Mater. 433, 431448.

10. Billone, M.C., Burtseva, T.A., and Liu, Y.Y., 2013, "Baseline Properties and DBTT of High-Burnup PWR Cladding Alloys," Proceeding of PATRAM 2013, San Francisco, CA.

11. Large, W.S. and Sindelar, R.L., 1997, "Review of Drying Methods for Spent Nuclear Fuel," Westinghouse Savannah River Company, Savannah River Site, Aiken, SC, report number WSRC-TR0075

12. Kim, H. G. Jeong, Y. H. and Kim, K. T., 2010, "The effects of creep and hydride on spent fuel integrity during interim dry storage," Nuclear Engineering and Technology, vol.42, no.3.

13. Bracey, W. and Smith, P., 2012, "Transnuclear Drying Experience and Lessons Learned," ASTM Workshop, Atlanta, GA.

14. Schaaf, S.A. and Chambre, P.L, 1961, Flow of Rarefied Gases, Princeton University Press, New Jersey.

15. Loeb, L.B., 1934, Kinetic Theory of Gases, $2^{\text {nd }}$ Edition, McGraw-Hill Book Company Inc.

16. Singh, K., 2012, "Method and apparatus for dehydrating high level waste based on dew point temperature measurement," Holtec International, Inc, Patent no.: US 8,266,823 B2.

17. Tseng, Y.S, Wang, J.R., Tsai, F.P., Cheng, Y.H., and Shih, C., 2011, "Thermal design investigation of a new tube-type dry-storage system through CFD simulations," Annals of Nuclear Energy, 38, 10881097.

18. Chalasani, N.R., Araya, P., and Greiner, M., 2009, "Benchmark of Computational Fluid Dynamics Simulations using Temperatures Measured within Enclosed Vertical and Horizontal Arrays of Heated Rods," Nuclear Technology., Vol. 167, No. 3, pp. 371-383.

19. Chalasani, N.R and Greiner, M., 2010, "Benchmark of Computational Fluid Dynamics Simulations Using Temperatures Measured Within Enclosed Vertical and Horizontal Arrays of Heated Rods," PVP2010-25803, Proceedings of the ASME 2010 Pressure Vessels and Piping Division/K-PVP Conference, Bellevue, Washington, USA.

20. Yoo, S.H., No, H.C., Kim, H. M., and Lee, E.H., 2010. "Full-scope simulation of a dry storage cask using computational fluid dynamics," Nuclear Engineering and Design, Vol. 240, pp. 4111-4122. 
21. Brewster, R.A., Baglietto, E., Volpenhein, E., and Bajwa, C.S., 2012, "CFD Analyses of the TN-24P PWR Spent Fuel Storage Cask," Proceeding of the ASME 2012 Pressure Vessels and Piping Conference, Toronto, ON, Canada.

22. Holtec International, 2000, "Topical Report on the HI-STAR/HI-STORM Thermal Model and its Benchmarking with Full-Scale Cask Test Data," Holtec Report No. HI-992252.

23. Pacific Northwest Laboratory, et al., 1987, "The TN-24P PWR Spent Fuel Storage Cask: Testing and Analyses," EPRI NP-5128.

24. TN 24P certificate of Compliance, "Certificate of compliance for spent fuel storage casks, NUHOMS ${ }^{\circledR}$ 24P," Certificate number 1004, Docket number 72-1004, 1/23/95.

25. Final Safety Analysis Report (FSAR), "Standardized advanced NUHOMS ${ }^{\circledR}$ horizontal modular storage system for irradiated nuclear fuel," Transnuclear, Inc, NUH-003, Revision 8, June 2004.

26. Graur, I., Ho., M.T. and Wüest, M., 2013, "Simulation of the transient heat transfer between two coaxial cylinders," J. Vac. Sci. Technol. A 31(6).

27. Graur, I.A., and Polikarpov, A., 2009, "Comparison of different kinetic models for the heat transfer problem". Heat and Mass Transfer, 46, pp. 237-244.

28. Lin, J. T. and Willis, D. R., 1972, "Kinetic Theory Analysis of Temperature Jump in a Polyatomic Gas," Phys. Fluids, vol. 15, p. 31.

29. Creer, J.M., Michener, T.E., McKinnon, M.A., Tanner, J.E., Gilbert, E.R. and Goodman, R.L., 1987, "The TN-24P PWR spent fuel storage cask: testing and analyses," Pacific Northwest National Laboratory (PNL), Interim Report PNL-6054.

30. Modest, M.F., 2003, Radiative Heat Transfer, $2^{\text {nd }}$ Edition, Academic Press, New York.

31. Transnuclear, Inc. "NUHOMS $®-24 \mathrm{PTH}$ DSC Thermal Evaluation for Storage and Transfer conditions," Project NO: NUH24PTH. Internal corresponding.

32. Bahney, R.H. and Lotz, T.L., 1996, "Spent Nuclear Fuel Effective Thermal Conductivity Report," Prepared for the U.S. DOE, Yucca Mountain Site Characterization Project Office by TRW Environmental Safety Systems, Inc.

33. Bird, G. A., 1983, "Definition of mean free path for real gases," Phys. Fluids, 26, 11.

34. Kennard, E. H., 1938, "Kinetic Theory of Gases," McGraw-Hill Book Company, Inc., New York.

35. Goodman, F. O., 1980. "Thermal accommodation coefficients," J. Phys. Chem. 84, 1431-144.

36. Saxena, S.C. and Joshi, R.K., 1989, "Thermal Accommodation \& Adsorption Coefficients of Gases," CRC Press.

37. Song, S., and Yovanovich, M. M., 1987, "Correlation of thermal accommodation coefficient for 'engineering' surfaces," Fundamentals of conduction and recent developments in contact resistance, Proceedings of the Twenty-fourth ASME National Heat Transfer Conference and Exhibition, Pittsburgh, PA, Aug. 9-12, pp. 107-116.

38. Sharipov, F., 2011, "Data on the Velocity Slip and Temperature Jump on a Gas-Solid Interface," J. Phys. Chem. Ref. Data 40, 023101.

39. Sharipov, F., 2004, "Data on the velocity slip and temperature jump coefficients," Thermal and Mechanical Simulation and Experiments in Micro-Electronics and Micro-Systems. Proc. 5th Int. Conf. EuroSimE, Belgium, pp. 243-249.

40. Maharjan, D., Hadj-Nacer, M., Ho, M.T., Stefanov, S.K., Graur, I. and Greiner, M., 2015, "Simulation of heat transfer across rarefied gas in annular and planar geometries: comparison of Navier-Stokes, Smodel and DSMC methods results," Proceeding of the International Conference on Nanochannels, \& Microchannels, and Minichannels (InterPACK/ICNMM2015), San Francisco, CA. 
(a)

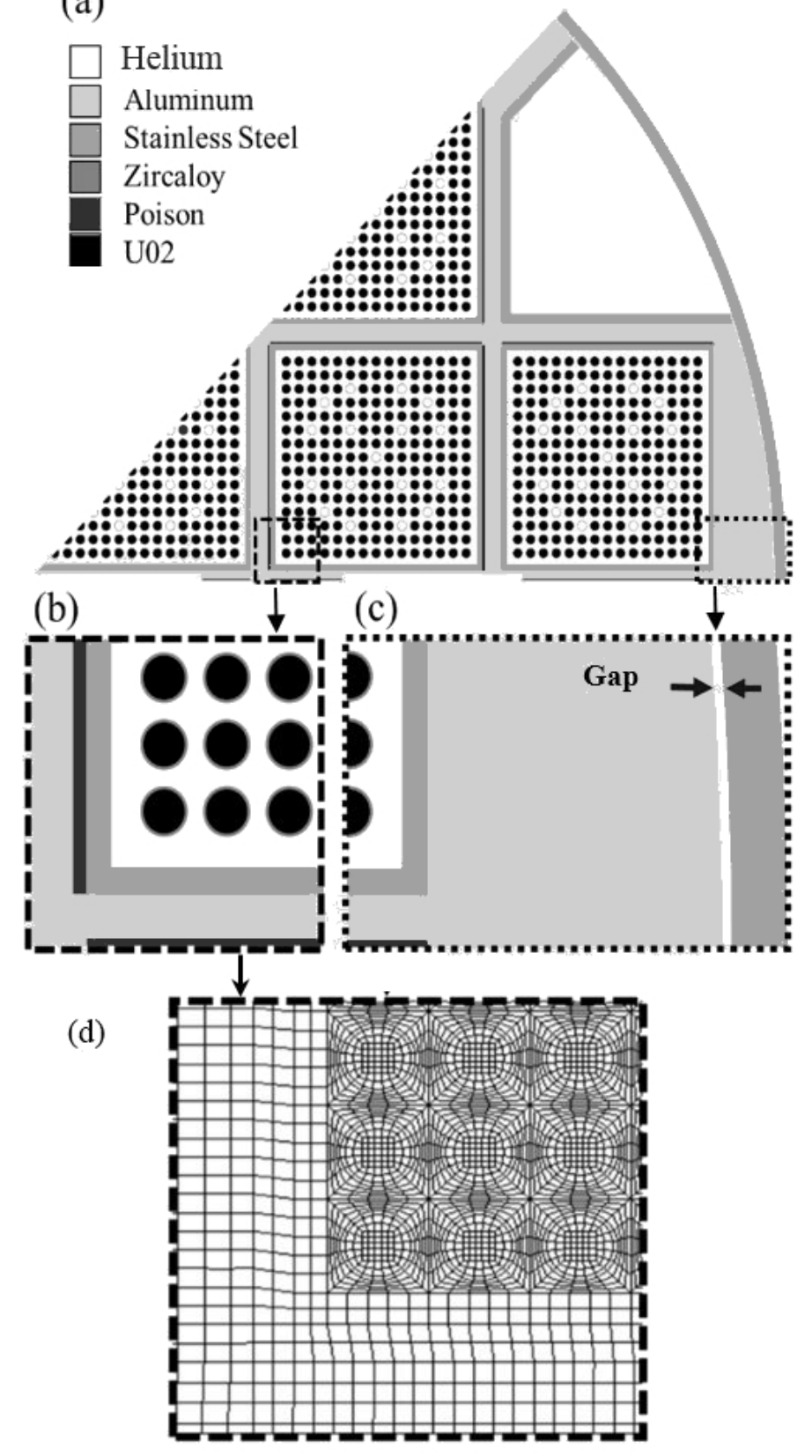

Figure 1. Two-dimensional model of one-eighth of a used nuclear fuel canister loaded with 24 PWR assemblies. (a) Gray-scale coded material regions. (b) Detail showing fuel rods, basket tubes BORAL ${ }^{\circledR}$ plates and aluminum structure. (c) Detail including gap between basket and canister. (d) Computational mesh for the region in part (b). 
Temperature

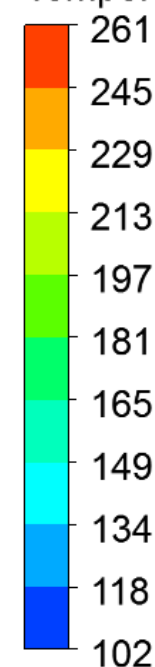

245

229

213

197

181

165

149

134

118

102
[C] 00000 2000000 10000000 00000000 00000000 00000000000 3000000000000 10000000000000 100000000000000

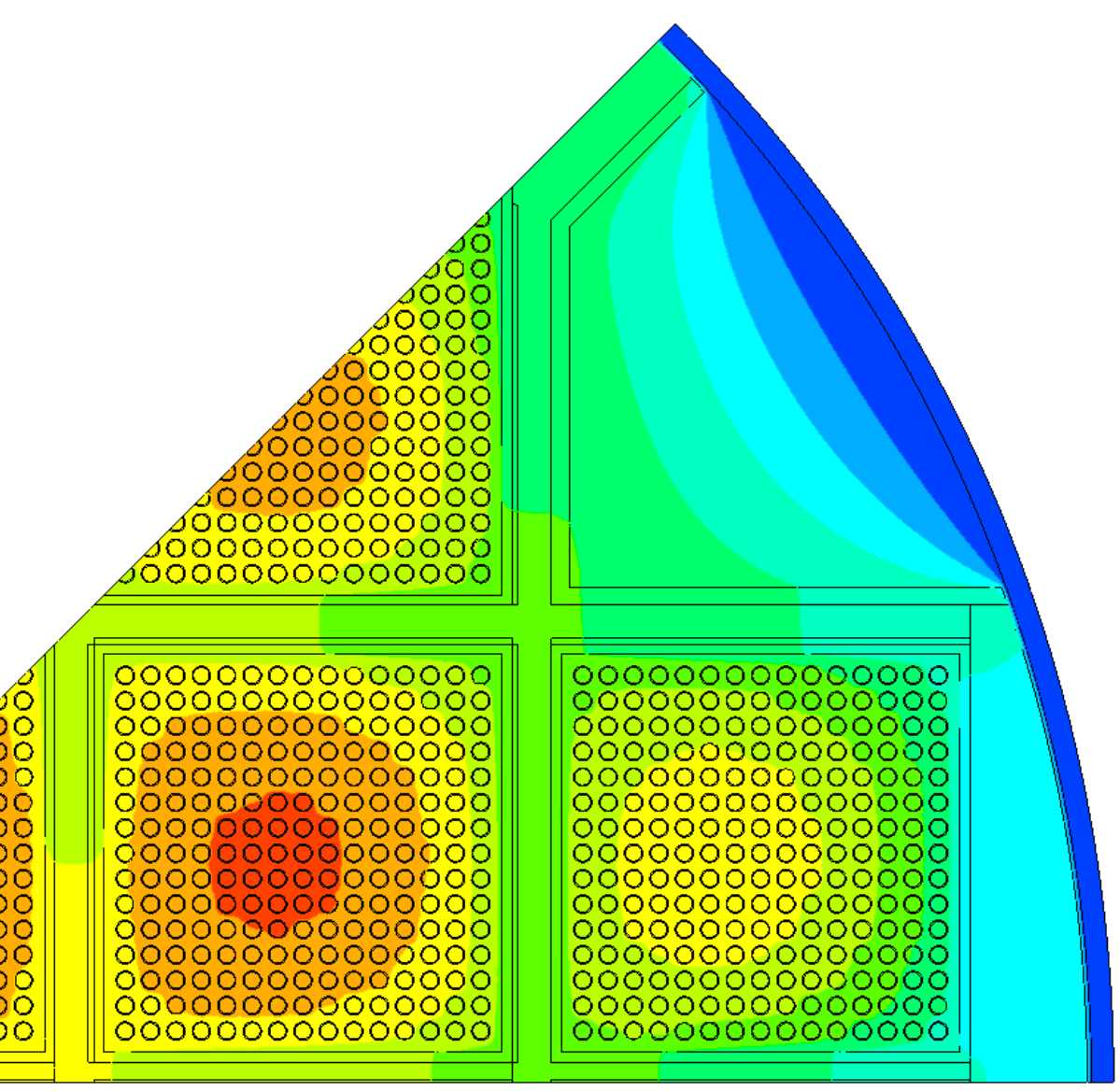

Figure 2. Loaded canister temperature contours for helium at $P=10^{5} \mathrm{~Pa}$ and $Q_{F}=1498 \mathrm{~W} /$ assembly. 


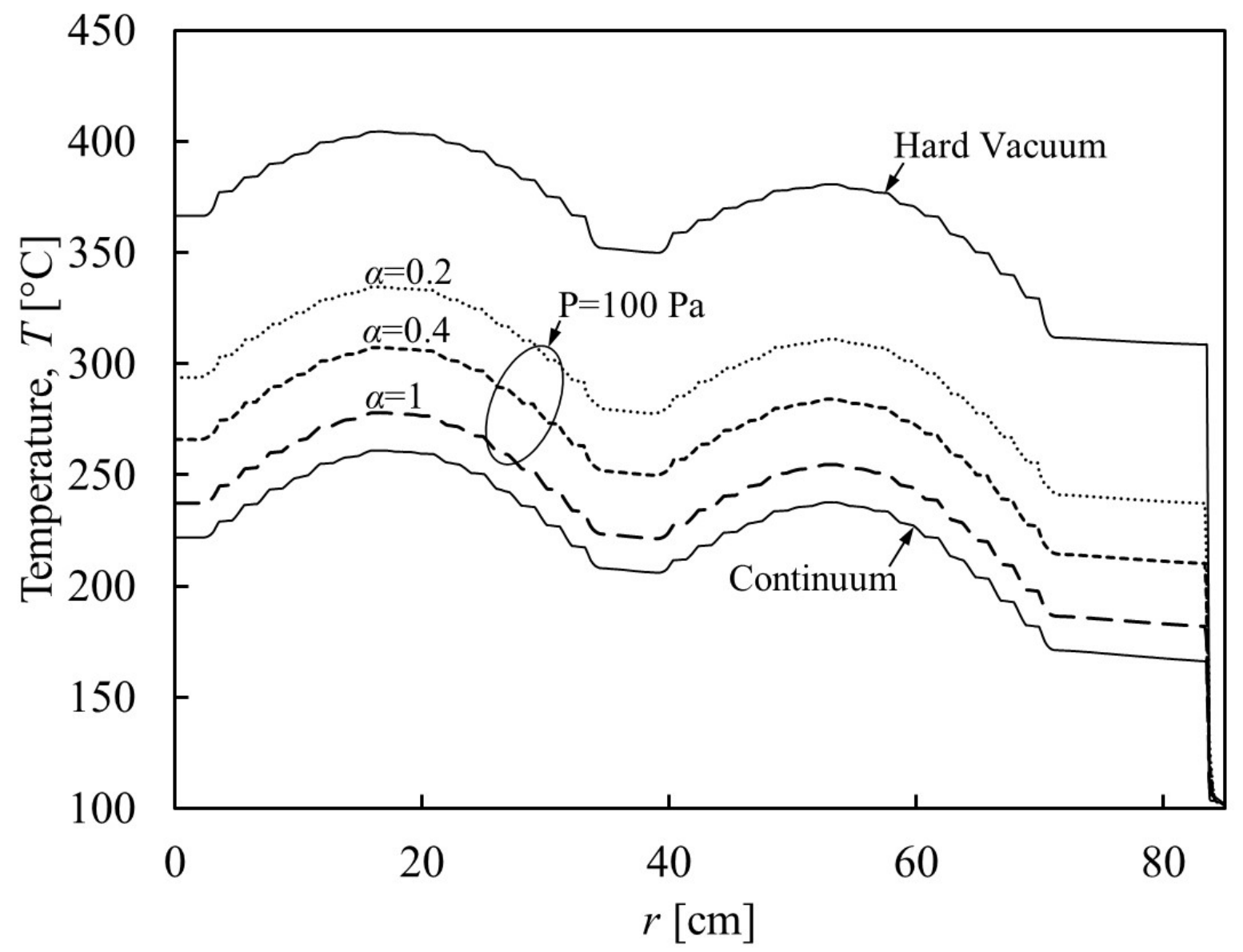

Figure 3. Temperature profiles along the $r$-axis shown in Fig. 2, for $Q_{F}=$ $1498 \mathrm{~W}$ /assembly. Results are shown for the Continuum model, the Temperature-Jump model at $P=100 \mathrm{~Pa}$ with three thermal accommodation coefficients, and a Hard Vacuum model. 
Table 1. Basket-surface and peak-clad temperature differences caused by reduced pressures, relative to continuum conditions.

\begin{tabular}{|c|c|c|c|}
\hline $\mathbf{P}[\mathbf{P a}]$ & $\boldsymbol{\alpha}$ & $\boldsymbol{\Delta} \boldsymbol{T}_{\boldsymbol{B S}}\left[{ }^{\circ} \mathbf{C}\right]$ & $\left.\boldsymbol{\Delta}_{\boldsymbol{P C}}{ }^{\circ}{ }^{\circ} \mathbf{C}\right]$ \\
\hline 0 & - & 142.4 & 143.5 \\
\hline \multirow{3}{*}{100} & 1 & 15.7 & 16.8 \\
\cline { 2 - 4 } & 0.4 & 44.1 & 46.3 \\
\cline { 2 - 4 } & 0.2 & 71.0 & 73.4 \\
\hline \multirow{3}{*}{400} & 1 & 4.2 & 4.6 \\
\cline { 2 - 4 } & 0.4 & 14.1 & 15.1 \\
\cline { 2 - 4 } & 0.2 & 27.9 & 29.6 \\
\hline
\end{tabular}




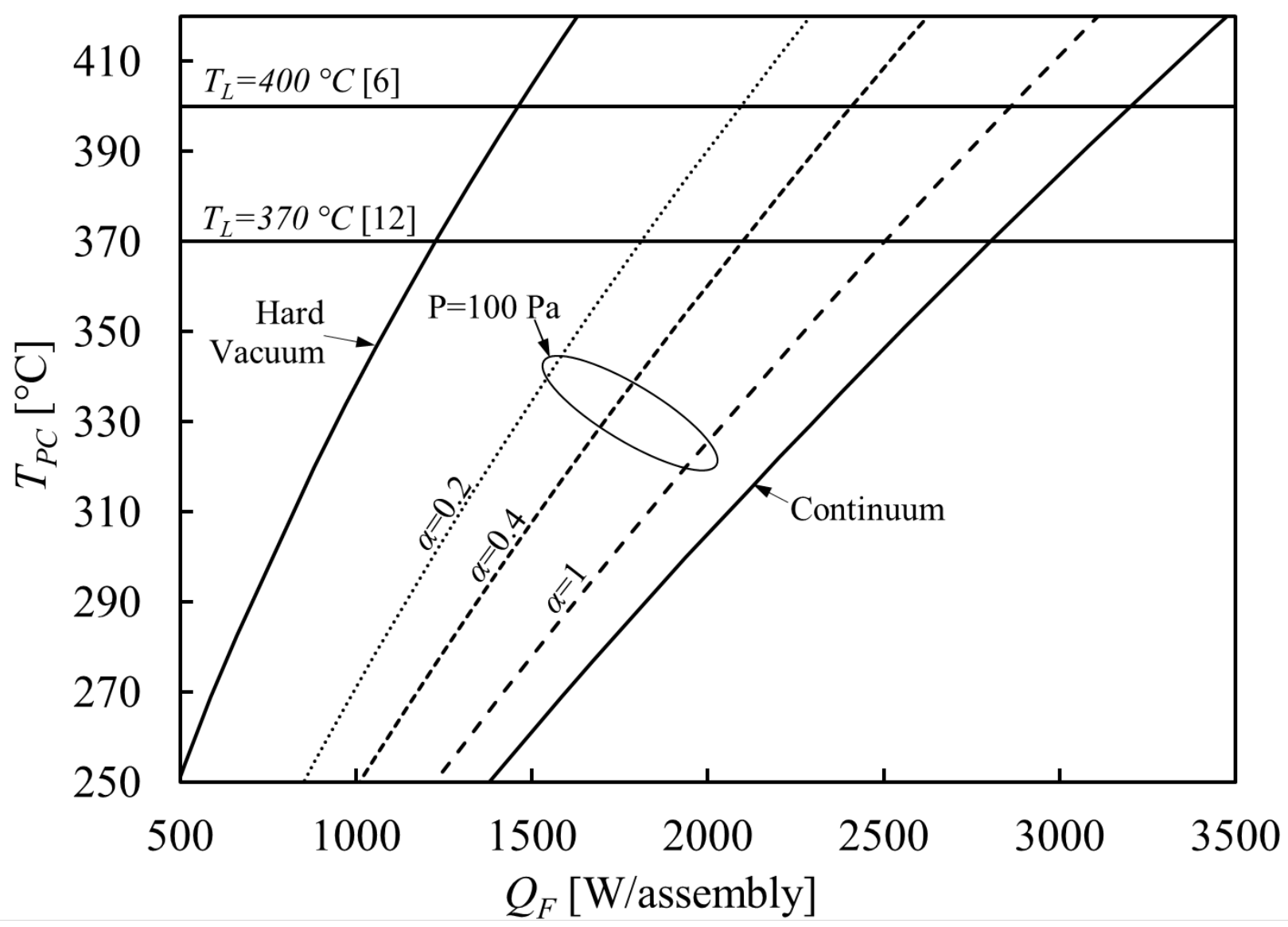

Figure 4. Peak clad temperature versus fuel heat generation rate for helium at $10^{5} \mathrm{~Pa}$ (Continuum model), $100 \mathrm{~Pa}$ (Temperature-Jump model) with three thermal accommodation coefficients, and $0 \mathrm{~Pa}$ (Hard Vacuum model). 
Table 2. Maximum allowable heat generation per assembly $Q_{F L}$ for helium at $10^{5} \mathrm{~Pa}$ (continuum model), 400 and $100 \mathrm{~Pa}$ (temperature-jump model) with three thermal accommodation coefficients, and $0 \mathrm{~Pa}$ (hard vacuum) for two cladding temperature limits.

\begin{tabular}{|c|c|c|c|c|c|c|c|c|}
\hline \multirow{2}{*}{$\begin{array}{c}\text { Cladding } \\
\text { Temperature }\end{array}$} & \multirow{2}{*}{$\begin{array}{c}\text { Continuum } \\
\text { Limit, } \boldsymbol{T}_{\boldsymbol{L}}\end{array}$} & $\boldsymbol{P}=\mathbf{1 0}^{\mathbf{5}} \mathbf{P a}$ & \multicolumn{5}{|c|}{ Rarefied } & \multirow{3}{*}{\begin{tabular}{c} 
Hard \\
Vacuum \\
\cline { 3 - 9 }
\end{tabular}} \\
\cline { 3 - 9 } & & $\boldsymbol{\alpha}=\mathbf{1}$ & $\boldsymbol{\alpha}=\mathbf{0 . 4}$ & $\boldsymbol{\alpha}=\mathbf{0 . 2}$ & $\boldsymbol{\alpha}=\mathbf{1}$ & $\boldsymbol{\alpha}=\mathbf{0 . 4}$ & $\boldsymbol{\alpha}=\mathbf{0 . 2}$ & $\boldsymbol{P}=\mathbf{0} \mathbf{P a}$ \\
\hline $\mathbf{4 0 0}^{\circ} \mathbf{C}$ & 3202 & 3101 & 2891 & 2645 & 2861 & 2408 & 2092 & 1461 \\
\hline $\mathbf{3 7 0}^{\circ} \mathbf{C}$ & 2804 & 2715 & 2529 & 2309 & 2502 & 2096 & 1810 & 1226 \\
\hline
\end{tabular}


(a)
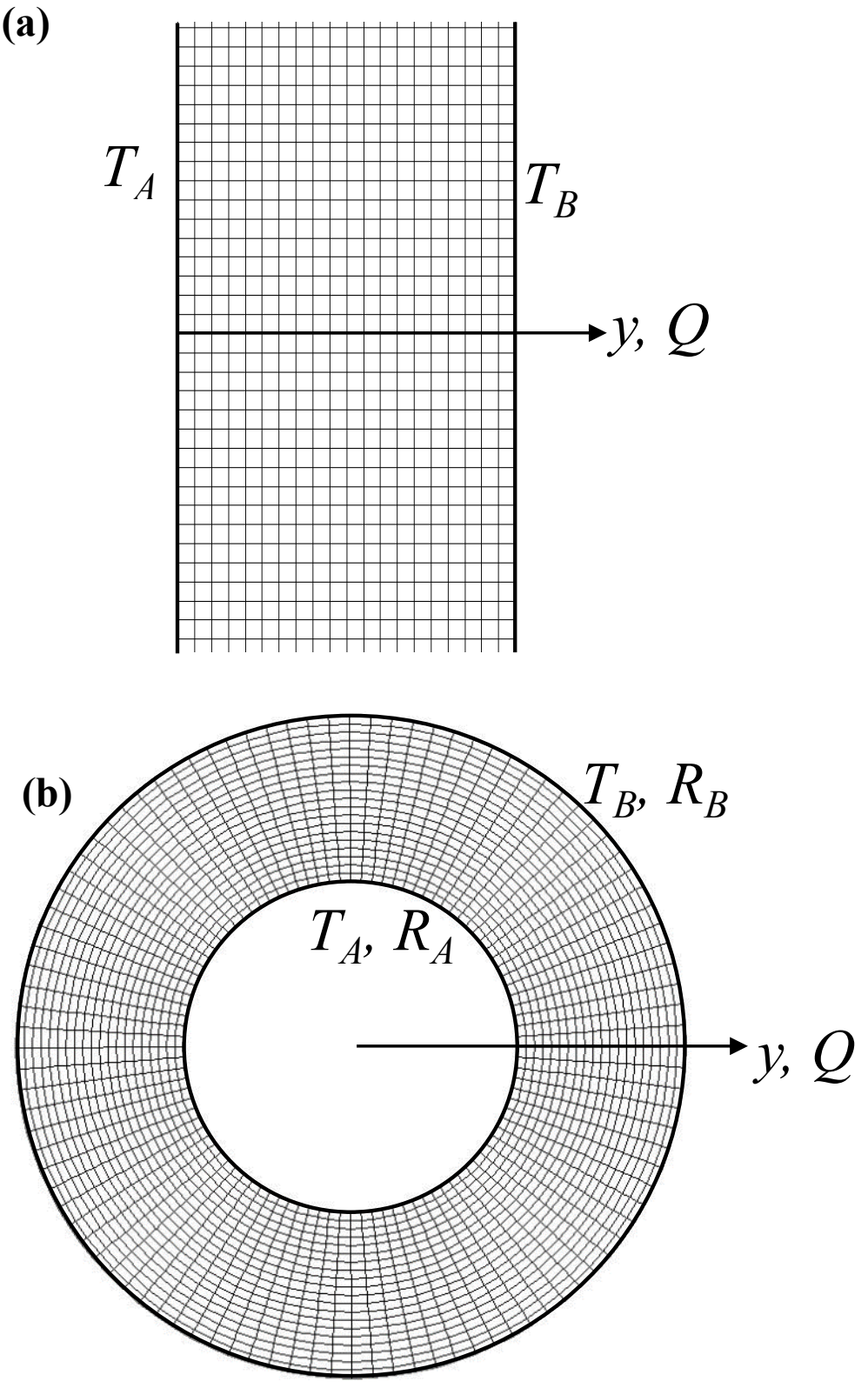

Figure 5. Domains used to benchmark Lin-Willis temperature-jump rarefied-gas heat-transfer model against $\mathrm{S}$-model kinetic equation, with $T_{A}=330 \mathrm{~K}$ and $T_{B}=300 \mathrm{~K}$. (a) Planar region between parallel plates (b) Annular region between concentric cylinders, with $R_{B} / R_{A}=2$. 

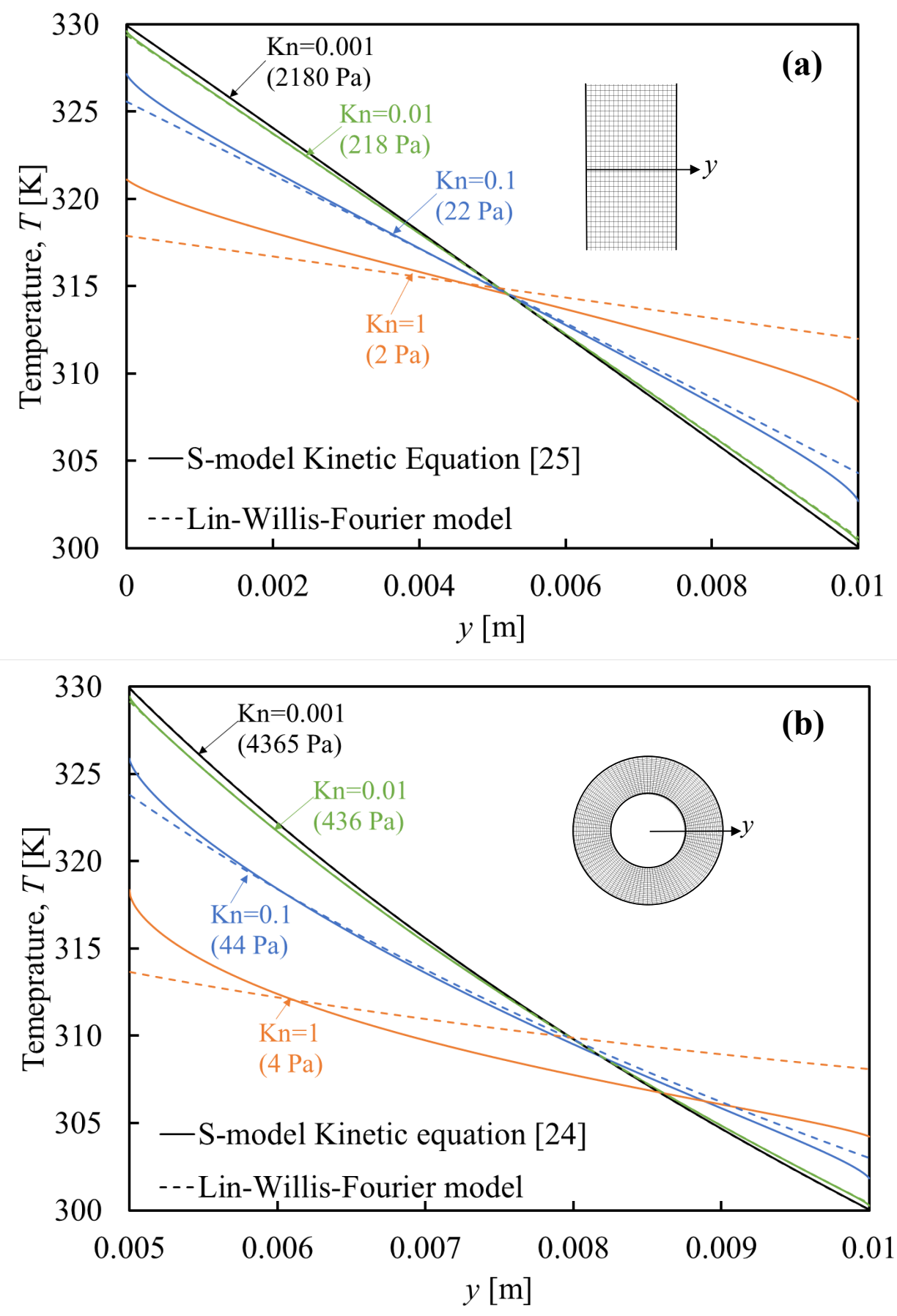

Figure 6. Temperature profiles within rarefied-helium-filled gaps with boundary temperatures of $300 \mathrm{~K}$ and $330 \mathrm{~K}$ using the S-model kinetic equation and the Lin-Willis model. (a) Planar gap. (b) Annular gap. 

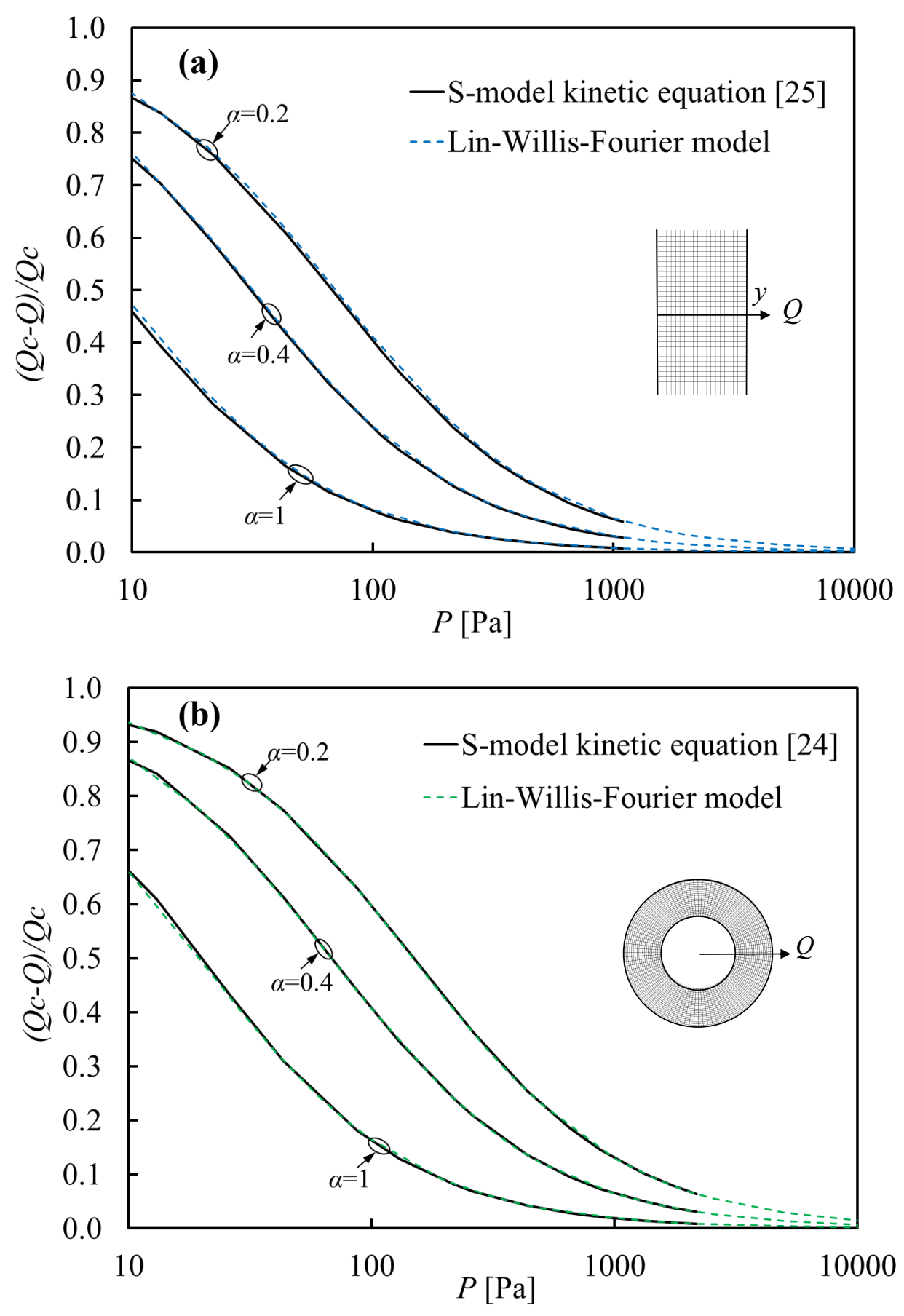

Figure 7. Heat transfer reduction ratio versus gas pressure and thermal accommodation coefficient from the S-model kinetic equation and the Lin-Willis temperature jump model. (a) Planar gap. (b) Annular gap. 


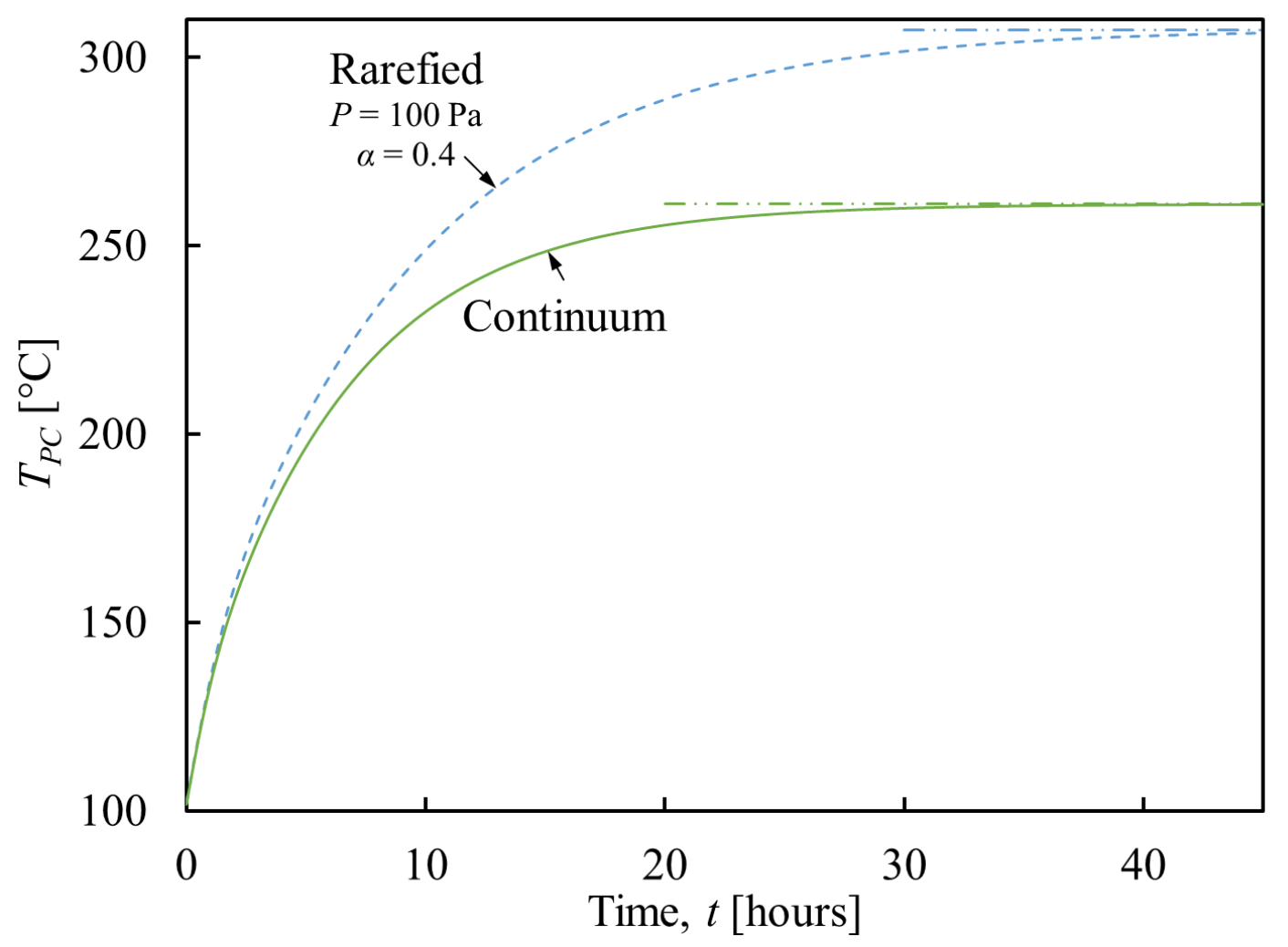

Figure 8. Peak clad temperature versus time after helium replaces water in the canister. Results are shown from the continuum model and the LinWillis rarefied model with $P=100 \mathrm{~Pa}$ and $\alpha=0.4$, for the respective fuel heat generation limits. 